\title{
Facial Asymmetry in Frequency Domain: The "Phase" Connection
}

\author{
Sinjini Mitra ${ }^{1}$, Marios Savvides ${ }^{2}$, and B.V.K. Vijaya Kumar ${ }^{2}$ \\ 1 Department of Statistics, Carnegie Mellon University, \\ Pittsburgh, PA 15213 \\ smitra@stat.cmu.edu \\ 2 Electrical and Computer Engineering Department, \\ Carnegie Mellon University, \\ Pittsburgh, PA 15213 \\ msavvid@cs.cmu.edu, kumar@ece.cmu.edu
}

\begin{abstract}
Facial asymmetry has now been established as a useful biometric for human identification in the presence of expression variations (1]). The current paper investigates an alternative representation of asymmetry in the frequency domain framework, and its significance in identification tasks in terms of the phase component of the frequency spectrum of an image. The importance of the latter in face reconstruction is well-known in the engineering literature ([2]) and this establishes a firm ground for the success of asymmetry as a potentially useful biometric. We also point out some useful implications of this connection and dual representation. Moreover, the frequency domain features are shown to be more robust to intra-personal distortions than the corresponding spatial measures and yield error rates as low as $4 \%$ on a dataset with images showing extreme expression variations.
\end{abstract}

\section{Introduction}

Human faces have two kinds of asymmetry - intrinsic and extrinsic. The former is caused by growth, injury and age-related changes, while the latter is affected by viewing orientation and lighting direction. We are however interested in intrinsic asymmetry which is directly related to the individual facial structure while extrinsic asymmetry can be controlled to a large extent or can be pre-processed or normalized. Psychologists say that the more asymmetric a face, the less attractive it is and more recognizable $([3,4])$. This indicates the potential significance of asymmetry in automatic face recognition problems.

A commonly accepted notion in computer vision is that human faces are bilaterally symmetric ([5]) and [6] reported no differences whatsoever in recognition rates while using only the right and left halves of the face. However, a well-known fact is that manifesting expressions cause a considerable amount of facial asymmetry, they being more intense on the left side of the face ([7]). Indeed [8] found differences in recognition rates for the two halves of the face under a given facial expression. 
Despite extensive studies on facial asymmetry, its use in human identification started in the computer vision community only in 2001 with the seminal work by Liu (9]), who for the first time showed that certain facial asymmetry measures are efficient human identification tools under expression variations. This was followed by more in-depth studies ([1], [10]) which further investigated the role and locations of different types of asymmetry measures both for human as well as expression classifications. But people have not so far utilized the frequency domain for developing facial asymmetry measures for recognition. This is a natural extension given that there exists much correspondence between the two domains. We explore this in depth in this paper.

The paper is organized as follows. Section 2 describes the dataset used and Section 3 introduces the new asymmetry measures in the frequency domain and presents some classification results. Section 4 describes the connection with Fourier domain phase and finally a discussion appears in Section 5.

\section{Data}

The dataset used here is a part of the "Cohn-Kanade AU-coded Facial Expression Database" ([1] ), consisting of images of 55 individuals expressing three different kinds of emotions - joy, anger and disgust. Each person was asked to express one emotion at a time by starting with a neutral expression and gradually evolving into its peak form. The data thus consists of video clips of people showing an emotion, each clip being broken down into several frames. The raw images are normalized using an affine transformation, the details being included in [1. Each normalized image is of size $128 \times 128$. Some normalized images from our database are shown in Fig. 1, This is the only available database for studying facial asymmetry and we use this smaller subset as out initial testbed.

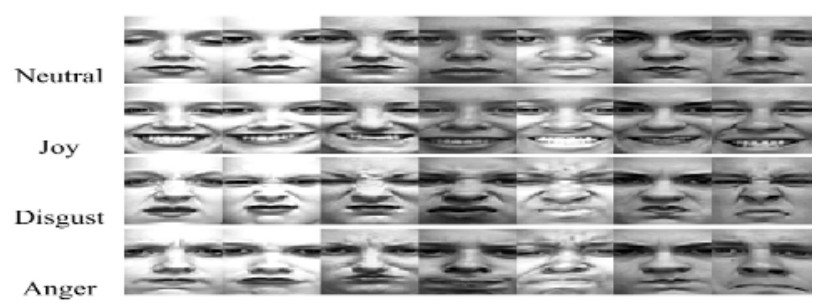

Fig. 1. Sample images from our database

\section{The Frequency Domain}

Many signal processing applications in computer engineering involve the frequency-domain representation of signals. The frequency spectrum consists of two components at each frequency: magnitude and phase. In 2D images 
particularly, the phase component captures more of the image intelligibility than magnitude and hence is very significant for performing image reconstruction ([2]). 12] showed that correlation filters built in the frequency domain can be used for efficient face-based recognition, and moreover, those based only on the phase component performed as well as the original filters ([13]). [14] demonstrated that performing PCA in the frequency domain by eliminating the magnitude spectrum not only outperformed spatial domain PCA, but also have attractive properties such as illumination and occlusion tolerance. These hence show that frequency domain features (particularly phase) possess the potential for improving classification results.

Symmetry properties of the Fourier transform are often very useful ([15]). Any sequence $x(n)$ can be expressed as a sum of an even part $x_{e}(n)$ and an odd part $x_{o}(n)$. Specifically, $x(n)=x_{e}(n)+x_{o}(n)$, where $x_{e}(n)=\frac{1}{2}(x(n)+x(-n))$ and $x_{o}(n)=\frac{1}{2}(x(n)-x(-n))$. Now, the even part actually corresponds to the symmetry part of the face and the odd part refers to the asymmetry. In other words, the more asymmetry a facial region possesses, the higher its value of the odd part and vice versa. Similarly, the more symmetric a particular face part is, the higher its value of the even part and vice versa. When a Fourier transform is performed on a real sequence $x(n)$, the even part $\left(x_{e}(n)\right)$ transforms to the real part of the Fourier transform and the odd part $\left(x_{o}(n)\right)$ transforms to its imaginary part (Fourier transform of any sequence is generally complex-valued). This implies that the asymmetry of the face (spatial domain) corresponds to the imaginary part of the Fourier transform and the symmetry part corresponds to the real part. This lays the ground for exploiting this correspondence between the two domains for developing more refined face identification tools based on asymmetry. Moreover, all these relations hold mainly for one-dimensional sequences alone, and hence we will define all our asymmetry features based on the Fourier transforms of row slices of the images.

\subsection{The Asymmetry Biometrics}

Following the notion presented in the earlier section, we define our asymmetry biometrics as:

- I-face: frequency-wise imaginary components of Fourier transforms of each row slice $-128 \times 128$ matrix of features

- Ave I-face: frequency-wise imaginary components of Fourier transforms of averages of two-row slices of the face $-64 \times 128$ matrix of features

- E-face: energy of the imaginary components of the Fourier transform of averages of two-row slices of the face - a feature vector of length 64

For all three sets of features, the higher their values the greater the amount of asymmetry, and vice versa. The averaging for E-faces is done in order to study if smoothing out noise in the image can reduce artificial asymmetry artifacts that gives misleading results. To the best of our knowledge, these frequency-based features as a means of representing facial asymmetry are fairly novel in any computer vision and pattern recognition problems. 


\subsection{Some Classification Results}

Of the various classifiers tried (including SVM, LDA, FF), the best results are obtained with Individual PCA (IPCA) which we report here. The IPCA method (13]) is different from the global PCA approach ([16]) where a subspace $W$ is computed from all the images regardless of identity. In individual PCA, on the other hand, subspaces $W_{p}$ are computed for each person $p$ and each test image is projected onto each individual subspace using $y_{p}=W_{p}^{T}\left(x-m_{p}\right)$. The reader is referred to [13] for more details about the procedure.

Table 1. Error rates for human identification using frequency-domain measures

\begin{tabular}{|c|c|c||c|c|}
\hline I-face & Ave I-face & E-face & Spatial D-face & D-face PCs \\
\hline $4.85 \%$ & $3.64 \%$ & $6.36 \%$ & $17.58 \%$ & $3.03 \%$ \\
\hline
\end{tabular}

The training is done on the neutral frames of the 55 individuals in the dataset and testing on the peak frames of the three emotions from all the people. Hence this represents an expression-invariant human identification problem, similar to the one reported in [1] which uses a simplistic measure of facial asymmetry in the spatial domain called D-face. The identification results in Table 1 show that our proposed frequency domain measures are significantly better than D-face ( $\mathrm{p}$ values $<0.0001$ ) and have no statistically significant differences with the $\mathrm{D}$-face PCs at the $1 \%$ level (p-values nearly 1 ). This shows quite convincingly that the frequency-domain measures are at least as robust to expression changes as their spatial domain counterparts. Further, the I-faces proved to be significantly better than the E-faces, which may be due to the loss of discriminative information caused by the feature reduction in the E-faces.

\section{Connection with Phase}

In this section we investigate in depth the correspondence of facial asymmetry with phase, that was briefly mentioned in Section 3. The symmetric part of a real sequence transforms to the real part in a Fourier transform whereas the asymmetric part transforms to the imaginary part. Thus the Fourier transform of a real symmetric sequence is real; that of a real asymmetric sequence is purely imaginary. Now, since phase is defined as $\theta=\tan ^{-1}\left(\frac{I}{R}\right)$, where $R$ and $I$ are respectively the real and the imaginary parts of the Fourier transform, $\theta=0$ in case the imaginary component $I$ is zero. In other words, a completely symmetric sequence gives rise to zero-phase frequency spectrum. In other words, absence of asymmetry implies zero phase, and vice versa. An important thing to note here is that all the above relations hold for $1 \mathrm{D}$ sequences $x(n)$ only (that is, when performing 1D Fourier transforms), as also pointed out in Section 3.

Interpreting these relations in terms of a face image, a completely symmetric face does not typically imply a zero-phase spectrum when applying a 2D Fourier transform. However, if we treat each row slice of the face as a $1 \mathrm{D}$ sequence and 
apply a 1D Fourier transform, the above relations will hold and each symmetric row will give rise to a $1 \mathrm{D}$ zero-phase spectrum. This is one primary reason we defined our asymmetry features the way we did. To make this study more rigorous, we constructed similar asymmetry features as before, using 1D row slices or averages of two-row slices but now based on "phase-only images" which have constant unit magnitude (obtained by dividing the Fourier transform by its magnitude). We call them I-face $\theta$, Ave I-face $_{\theta}$ and E-Face $\theta$.

Table 2. Error rates based on asymmetry features from phase-only images

\begin{tabular}{|c|c|c|}
\hline I-face $_{\theta}$ & Ave I-face $_{\theta}$ & E-face \\
\hline $4.85 \%$ & $5.45 \%$ & $7.27 \%$ \\
\hline
\end{tabular}

Table 2 shows the results from these phase-only features using the same experimental setup as before. They help establish, although empirically, an interesting connection between facial asymmetry and phase for human identification. The I-face features based on the actual images and the phase-only images produce exactly same classification results. This indicates that phase contains all the asymmetry of the original face, at least to the extent that is necessary for identification purposes, and no crucial information is lost by removing the magnitude. This leads us to believe that asymmetry may provide an alternative means of representing phase information. The Ave I-face $\theta$ and E-face $\theta$ results are different from Ave I-face and E-face (not significantly though) and this may be an artifact of averaging invloved in computing these features.

\section{Discussion}

We have shown in this paper that facial asymmetry measures in the frequency domain offer a promising potential as an useful biometric in practice, especially, in the presence of expression variations in face images. An error rate of less than $5 \%$ is impressive and desirable given that the test images are very different from the training ones. This in turn is very important for recognition routines in practice, for example, surveillance photos captured at airports are expected to be quite diverse with respect to facial expressions.

Moreover, our features are very simple and easy to compute, and they are based on well-known techniques such as Fourier transforms and phase which have well-established themselves as efficient face recognition tools in computer vision. However, the particular representations of them in terms of facial asymmetry that we consider in this paper are novel. It helped show that facial asymmetry, which has so far been treated only in the spatial domain, also has an analogous frequency domain version - a fact that can be utilized in signal processing applications too. More importantly, we have established an interesting connection between facial asymmetry and the phase component. Given the utter significance of phase in face-based identification, this helps in strengthening the 
scientific basis for the success of facial asymmetry in telling human beings apart. This relationship is a novel one and have not been explored earlier. It adds a whole new dimension to the concept of facial asymmetry and lays the ground for much further research in different directions. Particularly, the fact that asymmetry has an analogous spatial domain representation, provides a potential means of studying phase behavior in the image domain, and can be useful from several viewpoints such as, modeling, inference, etc.

Future research directions include investigating the role of the frequency domain features for expression classification, their tolerance to illumination changes, increasing the efficiency of the features, not only from classification point of view but also from storage and computational standpoints and application to larger and more diverse databases.

\section{References}

1. Liu, Y., Schmidt, K., Cohn, J., Mitra, S.: Facial asymmetry quantification for expression-invariant human identification. CVIU 91 (2003) 138-159

2. Hayes, M.H.: The reconstruction of a multidimensional sequence from the phase or magnitude of its fourier transform. ASSP 30 (1982) 140-154

3. Thornhill, R., Gangstad, S. W.: Facial attractiveness. Transactions in Cognitive Sciences 3 (1999) 452-460

4. Troje, N. F., Buelthoff, H. H.: How is bilateral symmetry of human faces used for recognition of novel views? Vision Research 38 (1998) 79-89

5. Seitz, S.M., Dyer, C.R.: View morphing. SIGGRAPH (1996) 21-30

6. Gutta, S., Philomin, V., Trajkovic, M.: An investigation into the use of partialfaces for face recognition. In: International Conference on Automatic Face and Gesture Recognition, Washington D.C. (2002) 33-38

7. Borod, J.D., Koff, E., Yecker, S., Santschi, C., Schmidt, J.M.: Facial asymmetry during emotional expression: gender, valence and measurement technique. Psychophysiology 36 (1998) 1209-1215

8. Martinez, A.M.: Recognizing imprecisely localized, partially occluded and expression variant faces from a single sample per class. PAMI 24 (2002) 748-763

9. Liu, Y., Schmidt, K., Cohn, J., Weaver, R.L.: Human facial asymmetry for expression-invariant facial identification. In: Automatic Face and Gesture Recognition. (2002)

10. Mitra, S., Liu, Y.: Local facial asymmetry for expression classification. In Proceedings of CVPR (2004)

11. Kanade, T., Cohn, J.F., Tian, Y.L.: Comprehensive database for facial expression analysis. In: Automatic Face and Gesture Recognition. (2000) 46-53

12. Savvides, M., Vijaya Kumar, B.V.K., Khosla, P.: Face verification using correlation filters. In: 3rd IEEE Automatic Identification Advanced Technologies, Tarrytown, NY (2002) 56-61

13. Savvides, M., Kumar, B.V.K.: Eigenphases vs.eigenfaces. ICPR (2004)

14. Savvides, M., Kumar, B.V.K., Khosla, P.K.: Corefaces - robust shift invariant PCA based correlation filter for illumination tolerant face recognition. CVPR (2004)

15. Oppenheim, A.V., Schafer, R.W.: Discrete-time Signal Processing. Prentice Hall, Englewood Cliffs, NJ (1989)

16. Turk, M.A., Pentland, A.P.: Face recognition using eigenfaces. In CVPR (1991) 